\title{
Existing models, but not neutrophil-to- lymphocyte ratio, are prognostic in malignant mesothelioma
}

\author{
T M Meniawy ${ }^{\star 1,2,3}$, J Creaney ${ }^{1,2}$, R A Lake ${ }^{1,2}$ and A K Nowak ${ }^{1,2,3}$ \\ ${ }^{1}$ M503, School of Medicine and Pharmacology, University of Western Australia, Sir Charles Gairdner Hospital, Nedlands, Western \\ Australia 6009, Australia; ${ }^{2}$ National Centre for Asbestos Related Disease, Sir Charles Gairdner Hospital, Nedlands, Western \\ Australia 6009, Australia and ${ }^{3}$ Department of Medical Oncology, Sir Charles Gairdner Hospital, Nedlands, Western Australia 6009, \\ Australia
}

Background: Recent studies proposed neutrophil-to-lymphocyte ratio (NLR) as a prognostic biomarker in malignant pleural mesothelioma (MPM). We examined baseline prognostic variables including NLR and the EORTC and CALGB models as predictors of overall survival (OS) in MPM.

Methods: In this retrospective study, 274 consecutive eligible, newly presenting patients with MPM were included. Of these, 159 received chemotherapy, 10 had tri-modality therapy, 2 underwent surgery only and 103 received supportive care alone. Univariate analyses and multivariate Cox models were calculated for OS.

Results: In univariate analysis, poor prognostic factors were: age $\geqslant 65$ years, nonepithelioid histology, stage III-IV, poor performance status (PS), weight loss, chest pain, low haemoglobin and high platelet count. A baseline NLR $\geqslant 5$ did not predict worse OS (hazard ratio (HR) 1.25; $P=0.122$ ). On multivariate analysis, age, histology, PS, weight loss, chest pain and platelet count remained significant. The EORTC and CALGB prognostic groups were validated as predictive for OS (HR 1.62; $P<0.001$ and HR 1.65; $P<0.001$, respectively).

Conclusion: Our findings validate standard prognostic variables and the existing EORTC and CALGB models, but not NLR, at initial diagnosis of MPM. In guiding patient management at diagnosis, it is important to consider multiple baseline variables that jointly predict survival.

Malignant pleural mesothelioma (MPM) is an aggressive neoplasm that is linked to exposure to asbestos. Its prognosis remains poor and the majority of patients ultimately succumb to their disease. The median survival of patients receiving current best treatment is 12 months (Vogelzang et al, 2003). A number of prognostic factors have been evaluated in multivariate analyses, and the best-known prognostic scoring systems have been proposed by the European Organisation for Research and Treatment of Cancer (EORTC) (Curran et al, 1998) and the Cancer and Leukaemia Group B (CALGB) (Herndon et al, 1998). The EORTC retrospectively evaluated prognostic variables in 204 patients from five phase II studies and found that performance status (PS), gender, white blood cell count (WCC), sarcomatous histology and a probable/ possible histologic diagnosis were independent prognostic factors. Patients were then classified into low- and high-risk groups with a 1 -year survival of $40 \%$ and $12 \%$, respectively. The CALGB index used regression trees to examine prognostic variables in 337 patients treated in seven phase II clinical trials. Six prognostic groups were identified based on age, performance status, haemoglobin $(\mathrm{Hb})$ level, WCC and the presence or absence of chest pain and weight loss. Both retrospective reports have been validated in subsequent studies (Edwards et al, 2000; 
Fennell et al, 2005), although only one validation was in consecutive presenting patients rather than a clinical trial population (Edwards et al, 2000).

Neutrophil-to-lymphocyte ratio (NLR), a marker of systemic inflammation, has been proposed as a prognostic factor from an analysis of a group of 173 patients undergoing systemic therapy (Kao et al, 2010). A baseline NLR of $<5$ was an independent predictor of better survival, and normalisation of NLR after one cycle of treatment was a predictor of improved survival on subgroup analysis. The prognostic value of NLR was subsequently demonstrated in four independent studies (Kao et al, 2011; Cedres et al, 2012; Pinato et al, 2012; Kao et al, 2013), although there were differences in study populations, and in two studies the NLR cutoffs used were different from the original threshold value of 5 . However, in one study of surgically treated patients, the association between NLR and survival was not statistically significant, although this study was underpowered for this analysis (Suzuki et al, 2011).

In this study, we explored prognostic variables at presentation in a cohort of consecutive, previously untreated patients with a diagnosis of MPM. Patients were included irrespective of subsequent treatment modality, including those who received only supportive care. Our aim was to validate the prognostic role of NLR as well as the EORTC and CALGB prognostic models for overall survival (OS) in MPM. There has been no previous validation of all three prognostic indicators in the same data set at initial presentation, the time point at which prognostic information is most important.

\section{MATERIALS AND METHODS}

Subjects. Consecutive patients with a diagnosis of MPM made between 1 January 2005 and 31 December 2010 who were diagnosed at or attended Sir Charles Gairdner Hospital, Nedlands, Western Australia, Australia, were included in this retrospective study. Selection criteria included availability of a neutrophil and lymphocyte count within 90 days of diagnosis, cytological or histologically confirmed MPM, absence of concurrent haematological malignancy and duration of follow-up $>90$ days from diagnosis for any censored patients.

Baseline prognostic clinical and laboratory variables were collected retrospectively from the clinical record. These included age, gender, histological subtype, Eastern Cooperative Oncology Group (ECOG) performance status, presence of chest pain or weight loss and treatment. Staging was determined according to the American Joint Committee on Cancer Staging System (AJCC), 7th Edition (Pleural Mesothelioma, 2010) according to surgical pathology (for extrapleural pneumonectomy (EPP) patients) or computerised tomography (CT) and, where performed, positron emission tomography (PET) imaging for all others. Laboratory variables at the time of diagnosis, before first and second cycles of chemotherapy and before commencing second-line chemotherapy, where applicable, were recorded. Variables to be assessed and cutoff determination were predetermined and categorised using the same cutoff points as the CALGB and EORTC models, where applicable. These included $\mathrm{Hb}$, which was expressed as the difference of patient $\mathrm{Hb}$ level relative to $160 \mathrm{gl}^{-1}$ in males and $140 \mathrm{gl}^{-1}$ in females ( $\mathrm{Hb}$ difference $<10 \mathrm{vs} \geqslant 10 \mathrm{gl}^{-1}$ ), WCC $\left(<8.3 v s \geqslant 8.3 \times 10^{9} 1^{-1}\right)$ (Curran et al, 1998), platelet count (PLT $<400 v s \geqslant 400 \times 10^{9} 1^{-1}$ ) (Herndon et al, 1998) and neutrophil and lymphocyte counts. The NLR was derived by dividing the absolute neutrophil count by the absolute lymphocyte count, and a cutoff of $<5 v s \geqslant 5$ was used in accordance with the first report of NLR in MPM (Kao et al, 2010).

The study was approved by the Sir Charles Gairdner Group Human Research Ethics Committee.
Statistical analyses. The primary end point of the study was OS, defined as the time from confirmed diagnosis of MPM to the date of death, or to the date of last follow-up for patients who have not died before the censor date.

A Cox regression power analysis was performed, and a sample size of 321 patients with an anticipated event rate of 0.85 was required to detect a HR of 1.5 as statistically significant with $\alpha=0.05$ and power of 0.80 on a covariate with the s.d. of 0.5. Baseline prognostic factors predictive for OS were entered into univariate Kaplan-Meier models and compared with the log-rank test. A Cox proportional hazards model was fitted to all individual prognostic variables to determine their independent effect, and diagnostics for the proportional hazards assumption were performed. Variables for EORTC, CALGB and the treatment groups were not entered into the Cox model as these are composite variables that are derived from, or determined by, other individual covariates in the model. Planned subgroup analyses of chemotherapy- or nonchemotherapy-treated groups for OS were performed for baseline NLR, and for NLR normalisation after one cycle of chemotherapy for patients with a prechemotherapy NLR $\geqslant 5$.

Missing values for prognostic variables were imputed using a multiple imputation by chained equation (MICE) approach (Van Buuren and Groothuis-Oudshoorn, 2011). Multivariate analyses were performed using the imputed data sets and repeated using data from patients with no missing values (complete case (CC) analysis).

Analyses were performed in $\mathrm{R}$ statistical computing software (R Core Team, 2013), SAS 9.3 (SAS Institute Inc., Cary, NC, USA) and GraphPad Prism version 6.0c (GraphPad Software, San Diego, CA, USA).

\section{RESULTS}

Patients. A total of 274 patients met the inclusion criteria and were included in the analyses. Of these, 169 (62\%) patients were treated with systemic chemotherapy, including 10 patients who underwent trimodality therapy (TMT); 103 (38\%) patients received supportive care alone (including palliative radiotherapy) and 2 patients underwent EPP but did not go on to receive TMT. For chemotherapy-treated patients, the median time from diagnosis to start of chemotherapy was 78 days (range 14 to 1658 days). A CONSORT diagram is shown in Figure 1.

At the census date of 15 May 2013, 251 patients were deceased, 18 patients were alive and 5 patients were censored ( 3 noncancerrelated deaths and 2 lost to follow-up), giving an event rate of 0.92 .

Median follow-up for patients who were alive was 45.5 months (range 29.0-88.3 months). The median OS from diagnosis for all patients $(n=274)$ was 13.3 months (95\% CI, 11.6-15.1 months). For chemotherapy-treated patients, median OS was 15.2 months (95\% CI, 13.8-17.9 months), whereas for the nonchemotherapytreated group it was 8.3 months (95\% CI, 5.7-11.1 months). For patients with a known chemotherapy start date $(n=163)$, the median OS from start of chemotherapy was 12.3 months, and was 11.7 months for those treated with chemotherapy only (i.e., nonsurgically, $n=153$ ).

The baseline distribution of patient demographics and disease characteristics according to treatment group are listed in Table 1; chemotherapy received is described in Table 2. Complete data for the 11 baseline prognostic variables analysed were available for $90 \%$ of patients. The mean duration between diagnosis and baseline blood results was 12 days (95\% CI, 10-14 days). In all patients NLR was available. Data were missing for staging in $6.6 \%$, weight loss in $3.3 \%$, chest pain in $2.9 \%$ and ECOG PS in $2.6 \%$ of patients. The CALGB prognostic group could not be calculated for $2.6 \%$ of patients, whereas the EORTC prognostic group was 


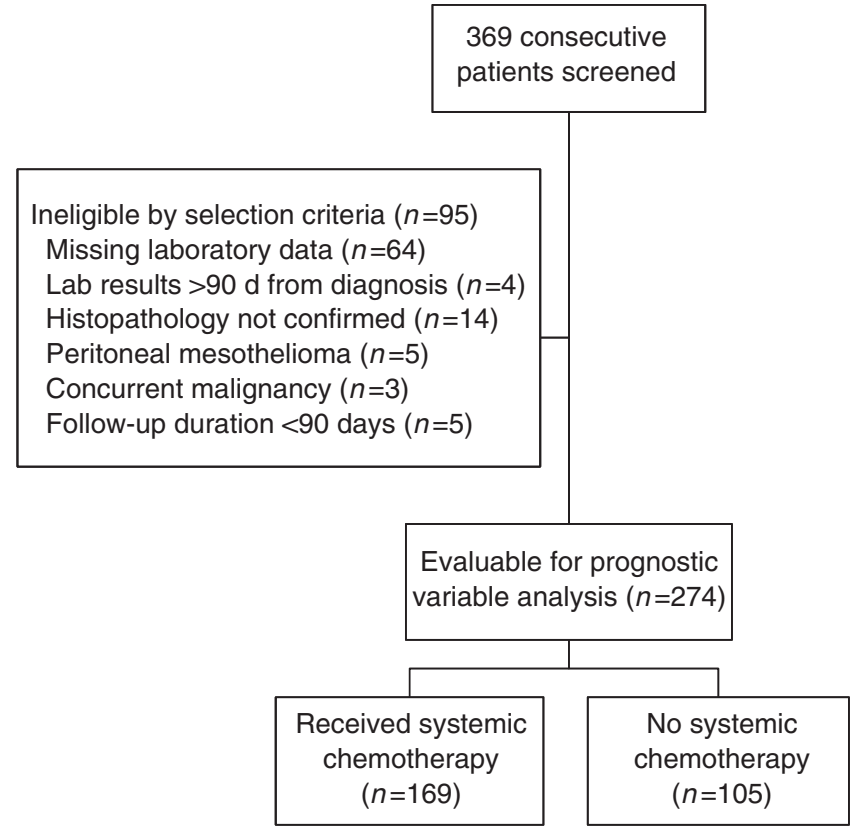

Figure 1. Study CONSORT diagram.

calculated for all patients, as the missing values would not have altered the prognostic risk group in those patients.

Univariate analyses. Results for the univariate analyses of 11 prespecified individual baseline variables are listed in Table 3. Variables that individually predicted for shorter OS were age $\geqslant 65$ years, nonepithelioid histology, AJCC stage III-IV, ECOG PS 2-3, weight loss, chest pain, $\mathrm{Hb}$ difference $\geqslant 10 \mathrm{gl}^{-1}$ and a platelet count $>400 \times 10^{9} 1^{-1}$. Both the EORTC and CALGB prognostic models were individually predictive for OS with a HR of 1.62 (95\% CI, 1.26-2.08; $P<0.001)$ and 1.65 (95\% CI, 1.36-1.99; $P<0.001$ ), respectively. A baseline NLR $\geqslant 5$ was not predictive for OS (HR 1.25; 95\% CI, 0.94-1.66; $P=0.122$; Figure 2).

Multivariate analyses. Individual variables analysed in univariate analyses were entered into the multivariate Cox model, irrespective of their significance. Analysis was performed using data from all study patients $(n=274)$ after multiple imputation (MI) of missing values, and revealed the following prognostic variables to be independent predictors of poor survival: age $\geqslant 65$ years, nonepithelioid histology, ECOG PS $2-3$, weight loss, chest pain and a baseline platelet count $>400 \times 10^{9} 1^{-1}$. Analysis using CC analysis revealed the same set of independent predictors as the imputed data sets (Table 3).

Preplanned subgroup analyses. Univariate analysis by chemotherapy subgroup showed that a baseline NLR of $\geqslant 5$ was not predictive of worse OS in chemotherapy-treated patients $(n=169)$ (HR, $1.08 ; 95 \% \mathrm{CI}, 0.74-1.58 ; P=0.69$ ) or in nonchemotherapytreated patients $(n=105)(\mathrm{HR}, 1.46 ; 95 \% \mathrm{CI}, 0.95-2.25 ; P=0.08)$. In chemotherapy-treated patients, $66(39 \%)$ had an NLR $>5$ at the time of commencement of chemotherapy that declined to $<5$ in 47 patients and remained elevated in 19 patients after one cycle of chemotherapy. Normalisation of NLR was individually prognostic for OS calculated from the time of commencing chemotherapy (HR, 2.2; 95\% CI, 1.25-4.03; $P<0.001$; Figure 3).

The post hoc analyses. The AJCC stage for patients with NLR $<5$ at diagnosis was I-II in $54 \%$ and III-IV in $46 \%$ of patients, whereas for patients with NLR > 5 it was I-II in $51 \%$ and III-IV in $49 \%$ of patients. Repeat measures of NLR at the time of diagnosis, at the time of commencement and after one cycle of chemotherapy were

\begin{tabular}{|c|c|c|c|c|c|c|}
\hline & & & \multicolumn{4}{|c|}{ Systemic chemotherapy } \\
\hline & \multicolumn{2}{|c|}{ All patients } & \multicolumn{2}{|c|}{ Yes } & \multicolumn{2}{|c|}{ No } \\
\hline & $\mathbf{N}$ & $\%$ & $\mathbf{N}$ & $\%$ & $\mathbf{N}$ & $\%$ \\
\hline \multicolumn{7}{|l|}{ Age } \\
\hline Median (range) & \multicolumn{2}{|c|}{$69(40-93)$} & \multicolumn{2}{|c|}{$66(40-80)$} & \multicolumn{2}{|c|}{75 (52-93) } \\
\hline \multicolumn{7}{|l|}{ Sex } \\
\hline Male & 237 & 86.5 & 143 & 84.6 & 94 & 89.5 \\
\hline Female & 37 & 13.5 & 26 & 15.4 & 11 & 10.5 \\
\hline \multicolumn{7}{|l|}{ Histopathology } \\
\hline Epithelioid & 115 & 42.0 & 68 & 40.2 & 47 & 44.8 \\
\hline Biphasic & 36 & 13.1 & 26 & 15.4 & 10 & 9.5 \\
\hline Sarcomatous & 32 & 11.7 & 19 & 11.2 & 13 & 12.4 \\
\hline Others & 91 & 33.2 & 56 & 33.1 & 35 & 33.3 \\
\hline \multicolumn{7}{|l|}{ AJCC stage } \\
\hline$|-| \mid$ & 137 & 50.0 & 79 & 46.7 & 58 & 55.2 \\
\hline III-IV & 119 & 43.4 & 86 & 50.9 & 33 & 31.4 \\
\hline Missing & 18 & 6.6 & 4 & 2.4 & 14 & 13.3 \\
\hline \multicolumn{7}{|c|}{ ECOG performance status } \\
\hline $0-1$ & 234 & 85.4 & 161 & 95.3 & 73 & 69.5 \\
\hline $2-3$ & 32 & 11.7 & 8 & 4.7 & 24 & 22.9 \\
\hline Missing & 8 & 2.9 & 0 & 0.0 & 8 & 7.6 \\
\hline \multicolumn{7}{|l|}{ Weight loss } \\
\hline Absent & 140 & 51.1 & 95 & 56.2 & 45 & 42.9 \\
\hline Present & 125 & 45.6 & 74 & 43.8 & 51 & 48.6 \\
\hline Missing & 9 & 3.3 & 0 & 0.0 & 9 & 8.6 \\
\hline \multicolumn{7}{|l|}{ Chest pain } \\
\hline Absent & 98 & 35.8 & 64 & 37.9 & 34 & 32.4 \\
\hline Present & 169 & 61.7 & 105 & 62.1 & 64 & 61.0 \\
\hline Missing & 7 & 2.6 & 0 & 0.0 & 7 & 6.7 \\
\hline \multicolumn{7}{|c|}{ EORTC prognostic group } \\
\hline Low risk & 135 & 49.3 & 81 & 47.9 & 54 & 51.4 \\
\hline High risk & 139 & 50.7 & 88 & 52.1 & 51 & 48.6 \\
\hline \multicolumn{7}{|c|}{ CALGB prognostic group } \\
\hline 1 or 2 & 56 & 20.4 & 34 & 20.1 & 22 & 21.0 \\
\hline 3 or 4 & 131 & 47.8 & 92 & 54.4 & 39 & 37.1 \\
\hline 5 or 6 & 80 & 29.2 & 43 & 25.4 & 37 & 35.2 \\
\hline Missing & 7 & 2.6 & 0 & 0.0 & 7 & 6.7 \\
\hline \multicolumn{7}{|c|}{ Neutrophil-to-lymphocyte ratio at baseline } \\
\hline Median (range) & \multicolumn{2}{|c|}{$3.53(1.28-22.6)$} & \multicolumn{2}{|c|}{$3.39(1.48-13.62)$} & \multicolumn{2}{|c|}{$3.77(1.28-22.6)$} \\
\hline$<5$ & 202 & 73.7 & 129 & 76.3 & 73 & 69.5 \\
\hline$\geqslant 5$ & 72 & 26.3 & 40 & 23.7 & 32 & 30.5 \\
\hline \multicolumn{7}{|c|}{ Overall survival from diagnosis } \\
\hline Median, months $(\mathrm{Cl})$ & \multicolumn{2}{|c|}{$13.3(11.6-15.1)$} & \multicolumn{2}{|c|}{$15.2(13.8-17.9)$} & \multicolumn{2}{|c|}{$8.3(5.7-11.1)$} \\
\hline $\begin{array}{l}\text { Abbreviations: } A J C C=a r \\
\text { cancer and leukemia gr } \\
\text { oncology group, EORTC } \\
N=\text { number. }\end{array}$ & $\begin{array}{l}\text { erican jo } \\
\text { pup } B ; C \\
=\text { europea }\end{array}$ & $\begin{array}{l}t \text { commit } \\
=\text { confider } \\
\text { organizat }\end{array}$ & $\begin{array}{l}\text { tee on } \\
\text { ce inter } \\
\text { ion for } r\end{array}$ & $\begin{array}{l}\text { er staging } \\
E C O G= \\
\text { arch and }\end{array}$ & $\begin{array}{l}\text { system } \\
\text { eastern } \\
\text { reatmen }\end{array}$ & $\begin{array}{l}\text { CALGB = } \\
\text { poperative } \\
\text { of cancer; }\end{array}$ \\
\hline
\end{tabular}


Table 2. Treatments received

\section{$\mathbf{N}$ \\ $\%$}

Chemotherapy group $(n=169)$, first-line regimens received

Cisplatin/pemetrexed

Carboplatin/pemetrexed

Cisplatin/gemcitabine

Carboplatin/gemcitabine

Gemcitabine

Unknown

Trimodality therapy

$>1$ line of chemotherapy received

Clinical trial participation (1st or 2 nd line)

72.2

$28 \quad 16.6$

$12 \quad 7.1$

$3 \quad 1.8$

$1 \quad 0.6$

$3 \quad 1.8$

$10 \quad 5.9$

$91-53.8$

$68 \quad 40.2$

No chemotherapy group $(n=105)$

Best supportive care

EPP and best supportive care

Abbreviation: EPP = extrapleural pneumonectomy

available for 157 out of 169 (93\%) chemotherapy-treated patients. The NLR was significantly different between the three time points $(P<0.001$; Figure 4). Median NLR was $3.39,4.44$ and 2.67 at diagnosis, prechemotherapy and after one cycle, respectively.

Exploratory univariate analyses of OS for NLR taken at diagnosis $(n=274)$ was performed at different NLR cutoff values of 3 , at the median value (3.53) and as a continuous variable, with no significant differences in survival revealed. These analyses were performed as proposed cutoffs used in the existing literature have been inconsistent (Kao et al, 2010, 2011; Pinato et al, 2012; Kao et al, 2013). For patients where a repeat NLR was available prechemotherapy $(n=163)$, exploratory univariate analysis of OS (calculated from start of chemotherapy) was performed at NLR cutoff points of 5, 3, and at the median value (4.44). Using an NLR cutoff of 5 prechemotherapy, HR was 1.39 (95\% CI, 1.00-1.94; $P=0.051)$, which remained nonsignificant on multivariate analysis $(P=0.08)$. No significant difference in survival was revealed for a cutoff of 3 or for the median value.

\section{DISCUSSION}

There has been considerable interest over the past decade in the association of systemic inflammatory markers and prognosis in both early and advanced cancer. A prognostic role for NLR in MPM was reported in a number of retrospective series, the largest of which are summarised in Table 4. The first report was a cohort of patients receiving systemic therapy (Kao et al, 2010), and further reports by the same group included surgically treated patients (Kao et al, 2011) and patients receiving compensation for asbestosrelated disease (Kao et al, 2013). Thus, NLR was proposed as a potential biomarker for stratification in clinical trials and for use in clinical practice. The primary aim of our study was to independently validate NLR as a prognostic marker in MPM and to further validate the EORTC and CALGB prognostic models, as well as known individual prognostic variables, all at the point of diagnosis and irrespective of subsequent treatment. Previous studies of NLR did not also examine the existing prognostic models; as such, the relative performance of NLR and the composite prognostic models was unknown.

Our study revealed that the baseline variables of advanced age $\geqslant 65$ years, nonepithelioid histology, poor PS, weight loss, chest pain and an elevated baseline platelet count were independently predictive of shorter survival, in keeping with the extensive body of prior literature of clinical prognostic factors in this disease. Both the EORTC and CALGB scores were individually predictive for OS with a highly statistically significant HR of 1.62 and 1.65, respectively. A baseline NLR of $\geqslant 5$ did not predict for shorter survival for the total cohort, or for any treatment modality group. For the subgroup of patients with an elevated prechemotherapy NLR $(n=69)$, normalisation of NLR to $<5$ after one cycle of chemotherapy was predictive, on univariate analysis, of longer survival, a finding that was consistent with a previous report (Kao et al, 2010). However, it is not known whether NLR normalisation would be an independent predictor if considered alongside other clinical parameters suggestive of response to chemotherapy such as improvement in PS, dyspnoea and chest pain, as these variables were not collected post chemotherapy. It is also unclear whether NLR normalisation would add to imaging information that is predictive of survival outcomes, such as CT and PET assessment after one or two treatment cycles (Byrne and Nowak, 2004; Francis et al, 2007).

Although our study validates the EORTC and CALGB models as well as other standard individual variables, the NLR finding was not consistent with previous series (Table 4). The strengths of the reported study include a large sample size, and the collection of relevant clinical variables, being the first NLR study to include chest pain and weight loss in addition to other standard variables, thus allowing the calculation of the CALGB prognostic group. The chemotherapy-treated patients in our study consistently received best standard chemotherapy regimens, with 165 out of 169 (98\%) patients receiving a first-line platinum doublet containing either pemetrexed or gemcitabine (Table 2). The cutoff values for NLR and other categorical variables used in our primary analyses were prespecified according to previous reports (Curran et al, 1998; Herndon et al, 1998; Kao et al, 2010), an important prerequisite for reliable validation. In previous reports, treatments were more heterogeneous and different cutoffs for NLR were used, which raises the possibility of data-driven cutoff optimisation (Table 4).

As we did not reproduce the prognostic importance of NLR, limited post hoc analyses were performed to explore the NLR findings of our study. Initiation of chemotherapy after initial diagnosis may be delayed according to the clinical scenario or patient preferences (median of 78 days in this study). We found that for 157 patients with a repeat NLR prechemotherapy, NLR increased significantly before subsequently declining after one cycle (Figure 4), and that a prechemotherapy NLR of $>5$ approached significance for predicting worse survival than NLR of $<5$ on univariate $(P=0.051)$ but not multivariate analysis $(P=0.08)$. This supports the hypothesis that systemic inflammatory markers, including NLR, increase as the disease progresses. However, a prognostic variable would be most useful in guiding decisions when considered at the time of diagnosis, rather than prechemotherapy when a treatment decision has already been made based on other clinical and imaging factors. We also explored NLR as a continuous variable, at a cutoff of 3 and at the median value, and no significant associations were revealed.

The role of systemic inflammation has been well documented in a number of malignancies, and has been linked to impaired nutritional status, poor PS and worse survival (Mcmillan, 2009). The absence of data on weight loss and performance status in previous reports may have exaggerated the relative significance of NLR, although we note that platelet count, which also reflects inflammation, was independently prognostic in our study.

Missing data is an inevitable and common problem in clinical studies and other types of data analyses, particularly in retrospective series; a number of methods to prevent and to handle missing data have been proposed (Ibrahim et al, 2012; Little and D'agostino, 2012). The most common method used in the literature to handle missing values is ignoring data for all patients 
A

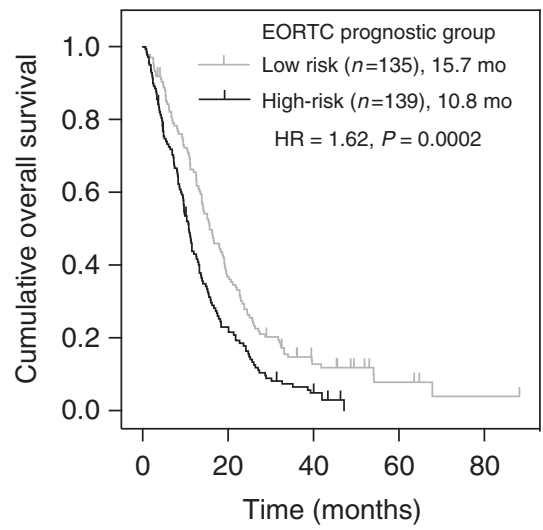

B

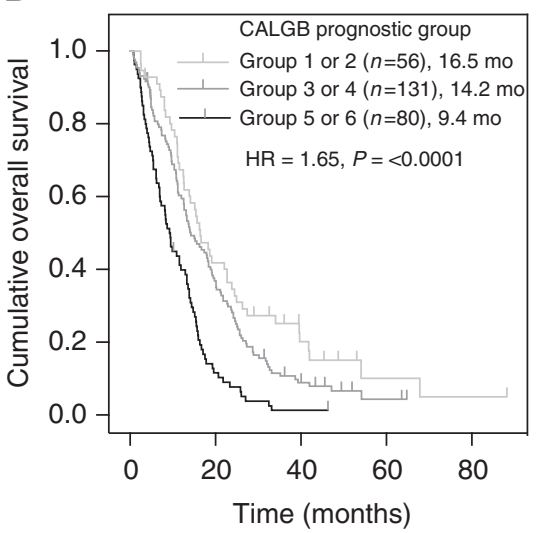

C

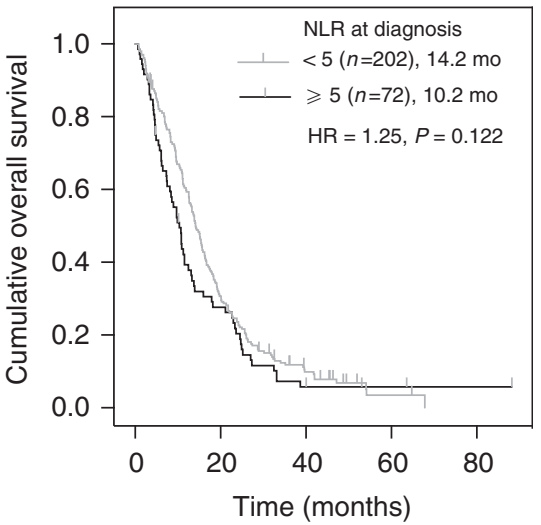

Figure 2. Univariate analyses of overall survival according to (A) EORTC prognostic group; (B) CALGB prognostic group; and (C) NLR. All at the time of diagnosis.

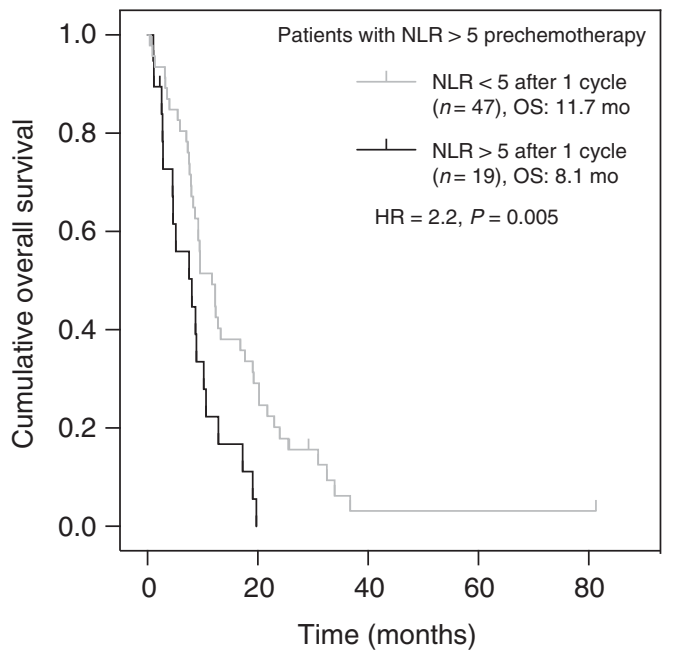

Figure 3. Univariate analyses of overall survival according to normalisation of NLR after one cycle of chemotherapy.

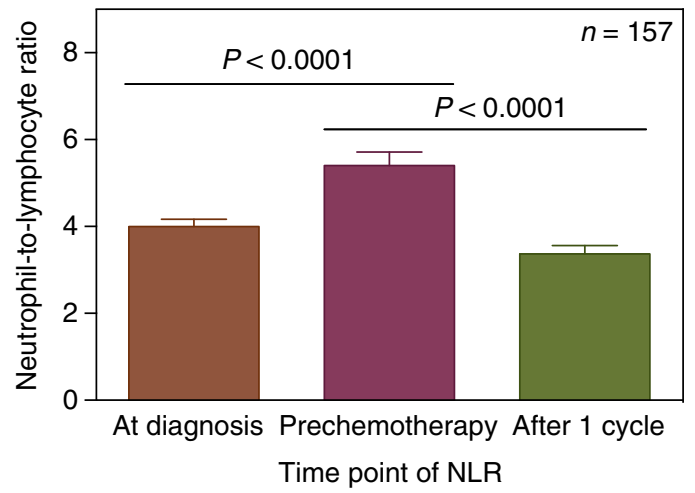

Figure 4. Neutrophil-to-lymphocyte ratio (mean and s.e.) at the time of diagnosis, prechemotherapy and after one cycle of treatment $(n=157)$.

collected, and results were similar to those revealed with CC analysis.

A large number of biomarkers have been proposed in oncology, with only a small number of clinically useful markers being validated despite hundreds of promising initial studies that were followed by studies failing to reproduce their results or revealing contradictory findings. This has been attributed to deficiencies in study design, analysis and inadequate reporting of findings. This prompted the development of the REMARK guidelines
(Guidelines for the REporting of tumor MARKer Studies), which aimed to encourage transparency in reporting of studies to contain sufficient information for readers to judge the usefulness and applicability of results and conclusions (Mcshane, 2005). One example given by the authors is the reporting of prognostic marker significance without disclosing extensive manipulation of data for cutoff optimisation and variable selection. Item 17 of the REMARK guidelines recommends that results should include 'an analysis in which the marker and standard prognostic variables are included, regardless of their statistical significance'. This is in contrast to the common practice of including in multivariate analyses only significant variables on univariate analysis. This report adheres to the REMARK guidelines where applicable, and clearly identifies prespecified methods and analyses, and the multivariate model was fit to variables significant in univariate analysis as well as clinically meaningful, standard prognostic variables.

The limitations of this study include its retrospective design and nonrandomised allocation of treatment groups, which prevents a comparison of the prognostic and predictive value of variables between treatment groups. Although patient screening was sequential, a selection bias is inherent when screened patients are excluded based on missing values for the prognostic markers being examined. It is possible that patients who were not fit for, or refused diagnostic testing, were lost to follow-up at an early point, or were diagnosed outside our tertiary institution in community or regional practices, and may have different covariate significance than our analysed sample, and hence results may not be generalisable to these populations. We also did not collect data on albumin (to determine the GPS) or CRP, and thus we could not include these markers of inflammation in this analysis. Finally, our study did not reach the original planned sample size because of a lower number of patients than estimated being seen between the dates we had ethical approval to span. Nevertheless, the higher than anticipated event rate $(0.92$ vs 0.85$)$ preserved the power of the study, and this remains the largest study of the prognostic value of NLR published to date. Despite the modest decrease in participant numbers from that planned, the CALGB and EORTC prognostic models nevertheless demonstrated significant prognostic value with hazard ratios that substantially outperformed NLR.

In conclusion, this study validates established prognostic factors but did not find NLR to be prognostic in this cohort of MPM patients. Previous positive findings may be a result of heterogeneity in patient populations, variable selection and cutoff points used in reports of NLR to date. The prospect of a simple blood test providing prognostic information was promising, but did not withstand the rigour of independent validation. Although there is a known relationship between inflammatory markers and disease stage, we hypothesise that it is unlikely that a ratio of neutrophils to 
Table 4. Published multivariate analyses of neutrophil-to-lymphocyte ratio in malignant mesothelioma

\begin{tabular}{|c|c|c|c|c|c|}
\hline & Kao et al (2010) & Kao et al (2011) & Pinato et al (2012) & Kao et al (2013) & Meniawy et al (this study) \\
\hline $\begin{array}{l}\text { Total no. of study patients } \\
\text { No. in multivariate model } \\
\text { No. with NLR available }\end{array}$ & $\begin{array}{c}173 \\
N R \\
168(97 \%)\end{array}$ & $\begin{array}{c}85 \\
N R \\
84(99 \%)\end{array}$ & $\begin{array}{c}171 \\
N R \\
159(94 \%)\end{array}$ & $\begin{array}{c}148 \\
130 \\
79(53 \%)\end{array}$ & $\begin{array}{c}274 \\
274 \\
274(100 \%)\end{array}$ \\
\hline Treatments received & $\begin{array}{l}\text { Chemotherapy } \\
\text { First line (69\%) } \\
\text { Second line (31\%) }\end{array}$ & $\begin{array}{c}\text { Extrapleural } \\
\text { pneumonectomy } \\
(E P P)\end{array}$ & $\begin{array}{l}\text { Chemotherapy (41\%) } \\
\text { Supportive care (42\%) } \\
\text { Unknown (17\%) }\end{array}$ & $\begin{array}{c}\text { Chemotherapy (53\%) } \\
\text { Radiotherapy (34\%) } \\
\text { EPP (5\%) }\end{array}$ & $\begin{array}{c}\text { Chemotherapy (62\%) } \\
\text { Supportive care (38\%) } \\
\text { EPP (1\%) }\end{array}$ \\
\hline $\begin{array}{l}\text { Median baseline NLR } \\
\text { Cutoff used in analysis }\end{array}$ & $\begin{array}{c}N R \\
<5 v s \geqslant 5\end{array}$ & $\begin{array}{c}3 \\
<3 \text { vs } \geqslant 3\end{array}$ & $\begin{array}{c}N R \\
<5 \text { vs } \geqslant 5\end{array}$ & $\begin{array}{c}3.5 \\
<3 \text { vs } \geqslant 3\end{array}$ & $\begin{array}{c}3.5 \\
<5 \text { vs } \geqslant 5\end{array}$ \\
\hline \multicolumn{6}{|c|}{ Prognostic variables entered into final multivariate model } \\
\hline $\begin{array}{l}\text { Age } \\
\text { Gender } \\
\text { Nonepithelioid histology } \\
\text { Sarcomatous histology } \\
\text { Stage } \\
\text { Performance status } \\
\text { Weight loss } \\
\text { Chest pain } \\
\text { Hb level } \\
\text { White cell count } \\
\text { Platelet count } \\
\text { Baseline NLR } \\
\text { Calretinin score } \\
\text { mGPS } \\
\text { Albumin, EPS, CRP, PLR } \\
\text { Treatments received }\end{array}$ & $\begin{array}{c}\text { NS } \\
\text { NS } \\
+ \\
\\
\text { NS }\end{array}$ & $\begin{array}{l}\text { NS } \\
\text { NS } \\
\text { NS }\end{array}$ & $\begin{array}{c}\text { NS } \\
+ \\
+ \\
+ \\
\text { NS }\end{array}$ & $\begin{array}{c}\text { NS } \\
\text { NS } \\
+ \\
+ \\
+ \\
\\
\\
+ \\
\text { NS } \\
\text { NS } \\
+ \\
+ \\
\\
\\
+\end{array}$ & $\begin{array}{c}+ \\
\text { NS } \\
+ \\
+ \\
\text { NS } \\
+ \\
+ \\
+ \\
\text { NS } \\
\text { NS } \\
+ \\
\text { NS }\end{array}$ \\
\hline
\end{tabular}

total lymphocyte can provide an informative 'snapshot' of the antitumour immune response, which involves a variety of immune cells, where lymphocytes alone have many phenotypes and differing functionalities. We conclude that clinical management and decision making in MPM will continue to rely on patient preferences and consideration of multiple prognostic variables that independently, but also jointly, predict survival.

Future research is likely to include more studies of prognostic markers in MPM, where there is an urgent need for biomarkers that predict response to treatments such as chemotherapy and immunotherapy, as the era of personalised therapy in other cancers has yet to be utilised in the management of MPM. We suggest that researchers consider the most appropriate methods to prevent and handle missing data to avoid the omission of valuable information, and to apply recommendations from reporting guidelines such as REMARK to ensure transparent and complete reporting of prognostic biomarkers studies, which will lead to realistic and reproducible results and conclusions, and aid readers in considering the findings and their generalisability.

\section{ACKNOWLEDGEMENTS}

We are grateful to Dr Ian Dick for his assistance with data analysis, to Anthony Robinson, Litany Looby and Henry Steer for their contribution to data collection, and to the National Centre for Asbestos Related Disease for providing financial support.

\section{CONFLICT OF INTEREST}

The authors declare no conflict of interest.

\section{REFERENCES}

Burton A, Altman DG (2004) Missing covariate data within cancer prognostic studies: a review of current reporting and proposed guidelines. $\mathrm{Br}$ J Cancer 91: 4-8.

Byrne MJ, Nowak AK (2004) Modified RECIST criteria for assessment of response in malignant pleural mesothelioma. Ann Oncol 15: 257-260.

Cedres S, Montero MA, Martinez P, Martinez A, Rodriguez-Freixinos V, Torrejon D, Gabaldon A, Salcedo M, Ramon YCS, Felip E (2012) Exploratory analysis of activation of PTEN-PI3K pathway and downstream proteins in malignant pleural mesothelioma (MPM). Lung Cancer 77: 192-198.

Curran D, Sahmoud T, Therasse P, Van Meerbeeck J, Postmus PE, Giaccone G (1998) Prognostic factors in patients with pleural mesothelioma: the European Organization for Research and Treatment of Cancer experience. J Clin Oncol 16: 145-152.

Edwards JG, Abrams KR, Leverment JN, Spyt TJ, Waller DA, O’byrne KJ (2000) Prognostic factors for malignant mesothelioma in 142 patients: validation of CALGB and EORTC prognostic scoring systems. Thorax 55: 731-735.

Fennell DA, Parmar A, Shamash J, Evans MT, Sheaff MT, Sylvester R, Dhaliwal K, Gower N, Steele J, Rudd R (2005) Statistical validation of the EORTC prognostic model for malignant pleural mesothelioma based on three consecutive phase II trials. J Clin Oncol 23: 184-189.

Francis RJ, Byrne MJ, Van Der Schaaf AA, Boucek JA, Nowak AK, Phillips M, Price R, Patrikeos AP, Musk AW, Millward MJ (2007) Early prediction of response to chemotherapy and survival in malignant pleural mesothelioma using a novel semiautomated 3-dimensional volume-based analysis of serial 18F-FDG PET scans. J Nucl Med 48: 1449-1458.

Herndon JE, Green MR, Chahinian AP, Corson JM, Suzuki Y, Vogelzang NJ (1998) Factors predictive of survival among 337 patients with mesothelioma treated between 1984 and 1994 by the Cancer and Leukemia Group B. Chest 113: 723-731. 
Ibrahim JG, Chu H, Chen M-H (2012) Missing data in clinical studies: issues and methods. J Clin Oncol 30: 3297-3303.

Kao SC, Klebe S, Henderson DW, Reid G, Chatfield M, Armstrong NJ, Yan TD, Vardy J, Clarke S, Van Zandwijk N, Mccaughan B (2011) Low calretinin expression and high neutrophil-to-lymphocyte ratio are poor prognostic factors in patients with malignant mesothelioma undergoing extrapleural pneumonectomy. J Thorac Oncol 6: 1923-1929.

Kao SC, Pavlakis N, Harvie R, Vardy JL, Boyer MJ, Van Zandwijk N, Clarke SJ (2010) High blood neutrophil-to-lymphocyte ratio is an indicator of poor prognosis in malignant mesothelioma patients undergoing systemic therapy. Clin Cancer Res 16: 5805-5813.

Kao SC, Vardy J, Chatfield M, Corte P, Pavlakis N, Clarke C, Van Zandwijk N, Clarke S (2013) Validation of prognostic factors in malignant pleural mesothelioma: a retrospective analysis of data from patients seeking compensation from the New South Wales Dust Diseases Board. Clin Lung Cancer 14: 70-77.

Little RJ, D'agostino R (2012) The prevention and treatment of missing data in clinical trials. N Engl J Med: 1355-1360.

Mcmillan DC (2009) Systemic inflammation, nutritional status and survival in patients with cancer. Curr Opin Clin Nutr Metab Care 12: 223-226.

Mcshane LM (2005) Reporting recommendations for tumor marker prognostic studies. J Clin Oncol 23: 9067-9072.

Pinato DJ, Mauri FA, Ramakrishnan R, Wahab L, Lloyd T, Sharma R (2012) Inflammation-based prognostic indices in malignant pleural mesothelioma. J Thorac Oncol 7: 587-594.
Pleural mesothelioma (2010) In AJCC Cancer Staging Manual, Edge SB, Byrd DR, Crompton CC, Fritz AG, Greene FL, Trotti A (eds). 7th edn. pp 271-277. Springer: New York, NY.

R Core Team (2013) R: A Language and Environment for Statistical Computing. R Foundation for Statistical Computing: Vienna, Austria.

Schafer J (1977) Analysis of Incomplete Multivariate Data. Chapman \& Hall: London.

Suzuki K, Kadota K, Sima CS, Sadelain M, Rusch VW, Travis WD, Adusumilli PS (2011) Chronic inflammation in tumor stroma is an independent predictor of prolonged survival in epithelioid malignant pleural mesothelioma patients. Cancer Immunol Immunother 60: $1721-1728$.

Van Buuren S, Groothuis-Oudshoorn K (2011) mice: Multivariate Imputation by Chained Equations in R. J Stat Softw 45: 1-67.

Vogelzang NJ, Rusthoven JJ, Symanowski J, Denham C, Kaukel E, Ruffie P, Gatzemeier U, Boyer M, Emri S, Manegold C, Niyikiza C, Paoletti P (2003) Phase III study of pemetrexed in combination with cisplatin versus cisplatin alone in patients with malignant pleural mesothelioma. J Clin Oncol 21: 2636-2644.

This work is published under the standard license to publish agreement. After 12 months the work will become freely available and the license terms will switch to a Creative Commons AttributionNonCommercial-Share Alike 3.0 Unported License. 